\title{
An impact of Croatian journals measured by citation analysis from SCI-expanded database in time span 1975-2001
}

\author{
MLADEN ANDREIS, ${ }^{a}$ MAJA JOKIĆb \\ a Institut Ruđer Bošković, Zagreb (Croatia) \\ ${ }^{\mathrm{b}}$ National and University Library Zagreb (Croatia)
}

The aim of this research is to gain an insight into international recognition of the STM (Science, Technology, and Medicine) Croatian journals measured by citations in SCI-expanded database. The sample for the research was a citation analysis of 142 journals in time span 1975-2001 for papers published in 1975-1998. More than $90 \%$ of those journals are not indexed by SCI-expanded. For the purpose of this research we introduced a new scientometric indicator Normalized number of Citations per 100 Papers (NCP) that allows us direct comparison of the journals from various categories $\left(N C P=100 \mathrm{C} / \mathrm{P} / \mathrm{IF}_{1989}\right)$. We chose the year 1989 as a mean value for time span 1975-2001.

By citation analysis we established the influence of errors on recognition of Croatian journals and their articles. Obtained results show that an article-to-article link is not found for $32 \%$ of cited items. The most frequent type of error is journal title, $37 \%$, which indicates that approximately one third of Croatian journals can not be found when searching by journal title only.

Some Croatian journals, even not indexed by SCI-expanded, showed relatively high rank in an impact, i.e. their NCP is higher than 100, and number of citations per paper is higher than 1 .

\section{Introduction}

Journal as a key conventional channel in science communication is an often used object in bibliometric research. The diversity of journal research aspects can be seen in example of two issues that celebrate the $50^{\text {th }}$ anniversary of The Journal of the

Received March 12, 2007

Address for correspondence:

MAJA JOKIĆ

National and University Library Zagreb, P.O. Box 550, Zagreb, Croatia

E-mail: mjokic@nsk.hr

$0138-9130 /$ US $\$ 20.00$

Copyright (C) 2008 Akadémiai Kiadó, Budapest

All rights reserved 
American Society for Information Science (JASIS), or in example of Scientometrics [SCHUBERT, 2002] and GLÄNZEL \& AL. [2002]. Regarding the research approach, articles involved in journal research and evaluation can be divided into two groups, objective and subjective [TURBAN \& AL., 2004]. The objective approach is based on citations and citation analyses (citation counts over a certain time period), "total cites", "immediacy index", "total articles", "cited half-life" and "impact factor". The subjective approach, also called perception analysis approach, solicits information from experts such as academic staff, deans, or departmental heads.

Citation-based ranking of journals, introduced by Gross \& Gross, in far 1927 is frequently used as an objective method of journal evaluation. Determination of the status or of the position of a journal in its environment, either in comparison to the subject field or in contexts of scientific environment of a university or a country, especially in example of small countries, is important for many reasons. According to NISONGER [1999], it is important for scientists to know where to send manuscripts, in order to be more available to the auditorium to whom it is dedicated. From the aspect of faculty or university evaluation it is not all the same in which journals their scientists publish. For libraries, journal evaluation and ranking is important for the purpose of collection planning and development, as well as it is important for journal editors to improve the quality of their journals.

Citation database, indices, WoS (Web of Science: SCI expanded, SSCI and A\&HCI) ISI /Thomson Scientific, and statistical databases originated from citation indices, JCR (Journal Citation Reports) and ESI (Essential Science Indicators), up to now, are recognized in whole scientific world as a main instrument in science evaluation. Considering that $\mathrm{WoS}$ is a very selective database and covers less than $10 \%$ of world scientific productivity, it is clear that most journals, especially from non-English speaking countries and small scientific communities, are not indexed in it. Consequently, they are not covered by JCR, and even less in ESI. An example of selectivity of mentioned databases can be seen in the sample of Croatian journals. At the moment 13 Croatian journals are indexed by WoS, 12 of them have an IF (indexed in $\mathrm{SCI}$ and $\mathrm{SSCI}) .13^{\text {th }}$ journal, International Journal of the Aesthetics and Sociology of Music, is indexed by A\&HCI, which has no instrument like JCR. According to the data from ESI database, ${ }^{1}$ which gathers data from WoS, in the category of the most cited journals in time span 1995-2005, in all subject fields we found only one Croatian journal, Croat Chem Acta, ranked 273 among the 303 most cited journals in the field of chemistry.

If we used only mentioned indicators in scientometric research, more than $95 \%$ of scientific productivity in national journals of non-English speaking countries would be omitted. Due to this fact it is questionable how much are those journals generally internationally relevant. For small countries, with low scientific productivity or so

${ }^{1}$ http://www.isinet.com/products/evaltools/esi/ (retrieved December 2005). 
called "periphery countries", it is important to continuously monitor the position of their journals in relevant scientific surrounding, even when they are not indexed by ISI/Thomson Scientific sources.

One of the opportunities for these countries is scientometric instrument development and monitoring of availability and impact of those journals in relevant conventional and less conventional sources: from the availability and accessibility in electronic format and indexing in relevant bibliographic databases, monitoring the usage via log analysis (reading, downloading etc.) to the measuring citations in conventional citation databases, as ISI/Thomson or in the national citation indices etc. [BROWN, 2003]. At the end of 2004 two new citation databases appeared; a commercial one Scopus, ${ }^{2}$ and an open source Google Scholar, ${ }^{3}$ with a different concept about the corpus of covered sources in comparison with the concept of WoS. New citation sources offer a more comprehensive picture of the impact of specific publication, on an international level, given their concepts of selection of publications are not based on Bradford's law of distribution, as in the case of WoS. Consequently, national journals covered by new citation sources have a better chance for international visibility and recognition.

One of the newest researches concerning the measurement to which extent is a journal internationally recognized, was conducted by BUELA-CASAL \& AL. [2006]. They claimed that it should be made clear that internationality per se is not to be equated with quality. It is quite possible that a non-global, non-international journal may publish the best, most advanced and innovative research. In their research they focused on the identification of a complete set of factors necessary to accurately define and measure internationality in relation to academic journal publishing.

\section{Croatian scientific journals}

Croatian scientific community with $6017 \mathrm{FTE}^{4}$ (Full Time Equivalent) or $0.14 \%$ FTE researchers in all scientific fields, compared to the total number of inhabitants, is a relatively small one. Their scientific productivity on international level is measurable by number of papers in the internationally recognized journals, indexed by WoS. However, one of the indicators of scientific productivity and scientific activity are journals and papers published in Croatia. At the moment, Croatia has approximately 220 journals in all scientific fields. It is difficult to establish the exact number of active national journals, as they have different periodicity, from once a year to twelve times yearly. It is not a rare occasion that a journal who predicted dynamic of two issues a year, appears as a double issue. The time span in appearance of a new issue for some journals can be

\footnotetext{
2 http://www.scopus.com/scopus/home.url (retrieved 24 November 2006).

${ }^{3}$ http://scholar.google.com/ (retrieved 24 November 2006).

${ }^{4}$ UNESCO Institut for Statistics. Statistical data FTE avalialbe on:

http://www.uis.unesco.org/TEMPLATE/html/Exceltables/science/R\&DTables.xls (retrived 31 October 2006).
} 
even two years. Publishers of almost all Croatian journals are academic institutions or professional associations, and all of them are financed by Croatian Ministry of Science, Education and Sport. There are exceptions of a few journals whose publishers are small private commercial companies, since the year 2000. Almost all of the journals still appear in a printed version, even though tendency to electronic versions is noticeable. National and University Library Zagreb started with a pilot project Full-text database of Croatian journals. ${ }^{5}$ The idea of the project is to organize and develop textual database for all Croatian scientific and professional journals to be available as an open source.

Given that we have a relatively big number of journals compared to relatively small scientific community, journal editors compete every year for the financing support of the Ministry of Science, Education and Sport to enable publishing. Ministry has its own criteria for journal evaluation, such as editorial board, peer-review, type of papers (original articles, review articles, short communication \& notes, and professional papers), coverage by relevant international bibliographic databases, especially by ISI databases. In order to be covered by the relevant international bibliographic databases, it is important to be readable, which means that it is important to publish in English. We observed linear increase rates for Croatian STM journals during the second half of 1990s and later tendency to publish increasingly in English instead of Croatian, similarly to the results obtained by LEYERSDORFF \& ZHOU [2005].

\section{Croatian journals indexed by WoS}

From the sample of 220 Croatian journals, with different frequency of publishing, from one to twelve issues yearly, and with the different starting years, currently 13 of them are indexed by WoS. More precisely, 10 Croatian journals are indexed by SCIExpanded: Chemical and Biochemical Engineering Quarterly, Croatian Medical Journal, Croatica Chemica Acta, Food Technology and Biotechnology, Mathematical Inequalities and Applications, Metalurgija, Neurologia Croatica, Periodicum biologorum, Strojarstvo and Tekstil; in SSCI database are indexed 2 journals: Collegium Antropologicum and Društvena istraživanja, and in A\&HC is indexed The International Review of Music Aesthetics and Sociology. If we presume that quoted journals represent the key Croatian journals, which is $5.9 \%$ of 220 journals, in that case this fact is basically in accordance to the selection policy of ISI that WoS represent the "core" of the world scientific journals. For a small, non-English speaking country as Croatia is, it is not reasonable to expect that it will be represented in WoS with more journals. In a comparison with Italian journals, according to UGOLINI \& CASILLI [2003] 73 of Italian journals were indexed by SCI, all of which were English-language, among 10 Croatian journals which are indexed by SCI-expanded, only 8 are English-language.

${ }^{5}$ http://www.nsk.hr/DigitalLib2c.aspx?id=457 (retrieved 24 November 2006). 
Given that the WoS is one of the most selective databases, for many journals even only to be indexed in it, is a matter of prestige, especially for "periphery countries". However, indexing by itself does not represent much, if we do not take a step further by monitoring citations of journals and papers. ISI calculates IF (Impact Factor) by measuring citations of indexed journals. Just to show an example of position and ranking of national journals indexed by WoS (SCI and SSCI) for 14 countries which recently started as a new member of EC, (more precisely 12 of them, and 2 countries who are negotiated to be part of EC), 3 countries are not represented by any journals in WoS (Lithuania, Cyprus and Malta), countries which are represented in WoS, from 1 to 6 journals were: Latvia 1, Bulgaria 2, Slovenia 4, Turkey 4, and Romania with 6 journals. Countries following by increasing number of indexed journals are Croatia 12, Slovakia 14, Hungary 18, Czech Republic 27 and Poland 37. IF of quoted journals, only in the case of Poland and Czech Republic (3 journals ), was equal to or higher than one, respectively the highest was 2.377 , for the year 2001 [JOKIĆ, 2003].

A fact that majority of mentioned journals, including Croatian journals, have an IF less than one, taking in consideration specificity of scientific fields, indicates that those journals however are not appertain to the group of the most prestige journals. Even, taking in consideration all specificity of ISI categories, majority of those journals are ranked in the third group on the IF scale.

Compared to other Croatian journals, Croatica Chemica Acta (CCA) is a Croatian journal indexed by SCI database at the very beginning (1974), with a stabile IF that is the highest among Croatian journals. According to SOMBATSOMPOP \& AL. [2002], average journal IF of the Thai academic journals in their work was 0.069 , suggesting that each article had an opportunity to be cited of $6.9 \%$. Journals with longer ages tended to have more opportunity to be cited, and had greater IF compared to those with shorter publishing period. According to ARAUJO RUIZ \& AL. [2005] foreign journals have a higher impact than national non-English journals.

SCI-expanded in time span 1975-1998 indexed 10 Croatian journals, 6 of them were included from 1994 to 1998. This fact could speak in favour of DANELL [2000] that the establishment of new journals can be seen as an expression of both growth and differentiation of science. For a small scientific community, in particular way, it is recognition of their contribution to whole scientific community.

\section{Objectives of the research}

Except the research about indexing in relevant bibliographic databases and occasionally research for a particular journals [SOROKIN, 1990; JOKIĆ, 1998; MARIČÍĆ \& AL., 2000; JOKIĆ, 2001; JOKIĆ \& AL., 2002; JOKIĆ \& SIROTIĆ, 2002] and researches led by ANDREIS [1998], in Croatia, until now we have not had systematic researches concerning the scientific productivity and citation analysis of Croatian journals. 
Therefore, in this research we tried to clarify questions concerning impact of STM Croatian journals by measuring citations which they received from journals indexed by SCI-expanded database for articles published in time span 1975-1998. More than 90\% of journals from our sample are not indexed by SCI-expanded, but possibility for their citation in WoS indexed journals exists. This fact was the starting point of our research. We tried to establish the position of Croatian journals, comparing the number of received citations by each paper. We will discuss the differences in number of received citations concerning the established errors in citing, and potential position of some of the Croatian journals, which are not indexed by SCI-expanded, but according to obtained results they could be a part of that corpus.

\section{Data and methods}

Sample for this research were citation analysis data extracted from SCI-expanded database for 142 Croatian journals in the time span 1975-2001 for articles published in 1975-1998. In mentioned time span some journals ceased and some emerged, especially in 1990s (see Table 2).

For each journal we counted a number of papers published per year and we analysed obtained citations in detail. Cited items are identified with six parameters: author's surname and initials, journal's title, volume, page, and publication year, which define the article-to-article link, necessary to identify cited item. The SCI-expanded database consists of two types of citations: citations of papers, registered as source items (i.e. all cited parameters match citing paper and the abbreviated journal's title is unified) and citations of articles (books, patents, papers etc.) that are (i) beyond the time scale of a database, (ii) not included as source items or (iii) contain one or more errors in six parameters that define related source item. Our sample consisted of more than $90 \%$ of journals which could be counted in categories (ii) and (iii).

Whereas not all of the six parameters that connect citing with cited article are searchable, the data are collected with simultaneous author and journal search. Highly cited articles by authors with frequent name are additionally searched by name and year in order to discover errors in a journal name. Each new journal "title" found by searching by particular author is then searched to find misspellings of author's name/initials and the process is repeated until all of the data are double checked. Besides, for all the journals an additional search based on English translations of journal name, as well as expected errors is performed. This search covers usual errors based on handwriting in earlier period, for example $\mathrm{a} \leftrightarrow \mathrm{o}, \mathrm{n} \leftrightarrow \mathrm{u}, 4 \leftrightarrow 9$, and typewriting in later period such as $\mathrm{a} \leftrightarrow \mathrm{s}, \mathrm{n} \leftrightarrow \mathrm{b}, 4 \leftrightarrow 5$.

To get a more objective picture we compared our results with the number of citations per papers for same or similar categories/fields according to data for 22 fields obtained by Essential Science Indicators (ESI) Thomson/ISI, 1995-2005, as well with 
IF's for 1989. The year 1989, which is approximately in the middle of the observed time span, is used for normalization of citations. Obtained ratio of 1995-2005 citations vs. 1989 IF data for most categories to which Croatian journals belong to, is about 6.4 (chemistry $=6.3$, geosciences $=6.7$, mathematics $=6.5$, pharmacy and pharmacology $=$ 6.1). This fact clearly shows that the category indicators do not change significantly during the time period and can be used simultaneously, to take into account large differences between various categories. Given that the ESI data contain citations per papers for only 22 fields and do not allow a more detailed classification of categories compared to JCR, the standard IF of categories are calculated from printed version of 1989 JCR $\left(\right.$ IF $\left._{1989}\right)$ [KLAIĆ \& ANDREIS, TO BE SUBMITTED]. In this research we classified 142 Croatian journals in 55 categories according to JCR Science Edition classification schema. The categories are listed in Table 1 with the number of journal titles, citations per 100 papers $(100 \mathrm{C} / \mathrm{P})$, due to the relatively small numbers.

It is well known that ISI categories differ by an order of magnitude. However, some of these categories are too wide and others too narrow [LEYDESDORFF \& ZHOU, 2005]. Also, field categories differ in IF values. For example biochemistry has IF between 3 and 4 , while engineering's IF is only about 0.3 , and journals are usually compared only within certain categories. In order to compare journals within different categories, a normalization of citation data to the IF of corresponding category is necessary. We introduced a new scientometric indicator Normalized Number of Citations per 100 Papers (NCP) that allows direct comparison of journals from various categories defined as:

$$
\mathrm{NCP}=\frac{100 \mathrm{C} / \mathrm{P}}{\mathrm{IF}_{1989}}
$$

For example, mathematical journal Glasnik Mat, having only 77.4 citations per 100 papers, normalized by the $\mathrm{IF}_{1989}$ turns to be $237.3\left(\mathrm{IF}_{1989}\right.$ for mathematics $\left.=0.397\right)$. The value of 201.1 citations per 100 papers for journal Acta Adriatica corresponds to $\mathrm{NCP}=0207.2$ due to the $\mathrm{IF}_{1989}$, close to 1.000 (0.971).

According to SOMBATSOMPOP \& AL. [2002], journals with older ages tended to have more opportunity to be cited, and were greater in impact factor value compared to those with younger ages. However, our results (see Table 2) show that journals starting later are ranked highly (8 and 9), while some other journals that ceased earlier, like Praxis Med is ranked lowly (135). 
M. ANDREIS \& M. JOKIĆ: An impact of Croatian journals measured by citation analysis

Table 1. Classification of Croatian journals according to the JCR scientific categories (1989), with citations per 100 papers and calculated Impact Factor for 1989 (IF 1989$)$

\begin{tabular}{|c|c|c|c|}
\hline & $\begin{array}{c}\text { Number of } \\
\text { journals }\end{array}$ & $\begin{array}{c}\text { Citation } \\
\text { per } 100 \text { papers }\end{array}$ & $\mathrm{IF}_{1989}$ \\
\hline AGRICULTURAL SCIENCES & 18 & 5.4 & 0.501 \\
\hline 1. agriculture & 8 & 4.4 & 0.378 \\
\hline 2. agriculture, dairy \& animal science & 2 & 5.2 & 0.668 \\
\hline 3. fisheries & 3 & 3.2 & 0.692 \\
\hline 4. forestry & 5 & 8.9 & 0.495 \\
\hline BIOLOGICAL SCIENCES & 13 & 54.7 & 2.090 \\
\hline 5. biology & 3 & 52.1 & 1.797 \\
\hline 6. entomology & 1 & 47.6 & 0.529 \\
\hline 7. marine $\&$ freshwater biology & 2 & 189.6 & 0.971 \\
\hline 8. ornithology & 2 & 8.5 & 0.705 \\
\hline 9. parasitology & 1 & 58.6 & 1.051 \\
\hline 10. plant sciences & 4 & 30.8 & 1.090 \\
\hline CHEMISTRY & 2 & 444.8 & 1.252 \\
\hline 11. chemistry & 1 & 557.3 & 1.359 \\
\hline 12. polymer science & 1 & 24.9 & 0.929 \\
\hline ENGINEERNIG & 31 & 12.6 & 0.557 \\
\hline 13. biotechnology \& applied microbiology & 1 & 142.8 & 1.114 \\
\hline 14. computer science, interdisciplinary applications & 3 & 13.7 & 0.553 \\
\hline 15. construction \& building technology & 1 & 12.1 & 0.255 \\
\hline 16. energy \& fuels & 4 & 1.5 & 0.429 \\
\hline 17. engineering & 4 & 0.4 & 0.285 \\
\hline 18. engineering, chemical & 3 & 20.8 & 0.595 \\
\hline 19. engineering, civil & 2 & 1.0 & 0.384 \\
\hline 20. engineering, electrical \& electronic & 1 & 1.7 & 0.634 \\
\hline 21. engineering, mechanical & 2 & 10.2 & 0.199 \\
\hline 22. food science \& technology & 3 & 24.1 & 0.648 \\
\hline 23. medical laboratory technology & 2 & 0.5 & 0.954 \\
\hline 24. metallurgy \& mining & 5 & 18.3 & 0.502 \\
\hline GEOSCIENCES & 12 & 25.3 & 1.122 \\
\hline 25. geography & 4 & 6.0 & 0.589 \\
\hline 26. geology & 3 & 31.6 & 1.144 \\
\hline 27. geosciences, interdisciplinary & 2 & 44.6 & 1.207 \\
\hline 28. meteorology \& atmospheric sciences & 2 & 36.5 & 1.571 \\
\hline 29. paleontology & 1 & 196.6 & 0.680 \\
\hline MATHEMATICS & 3 & 71.9 & 0.397 \\
\hline 30. mathematics & 2 & 71.9 & 0.326 \\
\hline 31. mathematics, applied & 1 & 71.4 & 0.462 \\
\hline
\end{tabular}


Table 1. (cont.)

\begin{tabular}{|c|c|c|c|}
\hline & $\begin{array}{c}\text { Number of } \\
\text { journals }\end{array}$ & $\begin{array}{c}\text { Citation } \\
\text { per } 100 \text { papers }\end{array}$ & $\mathrm{IF}_{1989}$ \\
\hline MEDICAL SCIENCES & $\mathbf{5 0}$ & 23.4 & 1.617 \\
\hline 32. dentistry, oral surgery $\&$ medicine & 1 & 11.8 & 0.718 \\
\hline 33. endocrinology \& metabolism & 1 & 20.6 & 2.270 \\
\hline 34. gastroenterology \& hepatology & 1 & 8.2 & 1.825 \\
\hline 35. medicine, general \& internal & 8 & 21.8 & 1.613 \\
\hline 36. medicine, miscellaneous & 4 & 12.0 & 0.587 \\
\hline 37. medicine, research \& experimental & 10 & 2.8 & 1.881 \\
\hline 38. neurosciences & 3 & 7.0 & 2.127 \\
\hline 39. obstetrics \& gynecology & 3 & 12.7 & 1.156 \\
\hline 40. oncology & 1 & 7.5 & 2.205 \\
\hline 41. ophthalmology & 1 & 5.7 & 1.072 \\
\hline 42. otorhinolaryngology & 2 & 2.2 & 0.505 \\
\hline 43. pediatrics & 3 & 4.2 & 0.988 \\
\hline 44. pharmacology \& pharmacy & 5 & 116.9 & 1.512 \\
\hline 45. psychiatry & 1 & 18.6 & 1.549 \\
\hline 46. public, environmental \& occupational health & 1 & 59.8 & 1.136 \\
\hline 47. rheumatology & 1 & 6.2 & 1.800 \\
\hline 48. substance abuse & 1 & 19.2 & 1.202 \\
\hline 49. veterinary sciences & 3 & 24.9 & 0.429 \\
\hline MATERIAL SCIENCE & 1 & 19.9 & 0.616 \\
\hline 50. materials science & 1 & 19.9 & 0.604 \\
\hline MULTIDISCIPLINARY SCIENCES & 4 & 12.0 & 4.225 \\
\hline 51. multidisciplinary sciences & 4 & 12.0 & 4.225 \\
\hline PHYSICS & 8 & 93.3 & 1.740 \\
\hline 52. astronomy \& astrophysics & 1 & 177.1 & 2.068 \\
\hline 53. crystallography & 2 & 29.1 & 0.964 \\
\hline 54. oceanography & 2 & 330.1 & 0.821 \\
\hline 55. physics & 3 & 66.4 & 2.105 \\
\hline
\end{tabular}

\section{Results and discussion}

The total of 72829 source items originating from 142 journals in the time span 1975-1998 were searched for citations in the time span 1975-2001. Three additional years, when an average paper reaches a maximum number of citations, are added to allow papers published in journals starting in the last observed years to be cited. The searches by journal title and author name resulted in 28412 citations of 10772 items that is about 0.4 citations per paper or about 2.6 citations per cited paper.

It should be mentioned that JCR classifies some journals in several categories, while SCI also includes four categories from social sciences (SSCI). The total of $4194 \mathrm{SCI}$ 
journals (1989) are classified in 125 categories as follows: 3284 journals (78\%) are in one category, 814 journals in two categories and 96 journals in three categories. However, in this analysis each journal is classified only in a single category, according to the subject of its papers, while journals covering more than three categories are considered as multidisciplinary. This classification is generally acceptable for all of the journals, although some of them, such as TRAVAUX ICSOBA (Travaux du Comite international pour l'etude des bauxites, de l'alumine et de l'aluminium) which consists of papers related to various fields, such as metallurgy and mining, geosciences, inorganic chemistry and chemical engineering, but it can not be considered as an multidisciplinary journal for it does not cover biology, medicine, agriculture and mathematics.

According to JCR classification scheme of scientific fields, Croatian journals cover just determined number of fields (see Table 1). This is expectable, given that a country with a relatively low scientific potential is not able to keep pace within all fields equally.

The field represented with the highest number of journals is medicine with all of its subfields, altogether 51 (35.9\%) journals. By decreasing number of journals follows engineering with all its subfields, from biotechnology and food technology to polymer science and chemical engineering, altogether 31 or $21.8 \%$. In third place are journals engaged in agricultural science, 19 or $13.4 \%$, followed by bioscience and geosciences, each of them by 13 or $9.1 \%$. Physics is represented with 8 or $5.6 \%$ journals; multidisciplinary journals represented 4 or $2.8 \%$; mathematics 3 or $2.1 \%$; chemistry with 2 or $1.4 \%$ and material sciences with 1 or $0.7 \%$.

Comparing just the quoted data with the results obtained by KozLOWSKI \& AL. [1999] and MUST [2006] concerning the Central and East European Countries, scientific productivity and impact by citations for source items indexed by SCI-expended, deviation is noticeable. Namely, there is a common understanding that CEEC countries are strong in fundamental research. Our further research concerning citation analysis and received average number of citations by fields, confirms that chemistry and physics are fields with the highest number of citations received (Table 1 and Table 2).

The category of agricultural science is represented with four fields, with approximately equally number of citations per paper (C/P). Although journal Ann Forestales (Table 2) is one of Croatian journals with the highest ratio $\mathrm{C} / \mathrm{P}$, its contribution of only $2.6 \%$ papers in the category, which received almost $58 \%$ of all citations in the category, does not influence essentially on the value of NCP.

If we compare results of average $\mathrm{C} / \mathrm{P}=0.054$ for Croatian journals in agricultural science with the world mean value according the ESI 1995-2005, 4.81 citations per papers, the difference is noticeable. To understand the differences it is important to take into account that Croatian journals in this category are not indexed by SCI-expanded and papers are written in Croatian. 
Table 2. Croatian journals in SCI-Expanded (1975-1998) ranked by NCP

\begin{tabular}{|c|c|c|c|c|c|}
\hline $\begin{array}{l}\text { Journal title } \\
(\text { category from Table } 1)^{1}\end{array}$ & NCP & $\begin{array}{l}\text { Number of } \\
\text { papers }(P)\end{array}$ & $\begin{array}{l}\text { Number of } \\
\text { citations }(\mathrm{C})\end{array}$ & $100 \mathrm{C} / \mathrm{P}$ & $\begin{array}{c}\text { Publishing } \\
\text { period }\end{array}$ \\
\hline 1. THALASSIA JUGOSL (54) & 486.2 & 356 & 1421 & 399.2 & $1975-1991$ \\
\hline 2. CROAT CHEM ACTA (11) & 410.1 & 1825 & 10170 & 557.3 & $1975 \longrightarrow$ \\
\hline 3. ANN FORESTALES (4) & 390.1 & 58 & 112 & 193.1 & $1975 \longrightarrow$ \\
\hline 4. PALAEONTOLOGIA JUGOS (29) & 289.0 & 29 & 57 & 196.6 & $1975-1991$ \\
\hline 5. GLASNIK MAT (30) & 237.3 & 875 & 677 & 77.4 & $1975 \longrightarrow$ \\
\hline 6. ACTA ADRIATICA (7) & 207.2 & 327 & 658 & 201.2 & $1975 \longrightarrow$ \\
\hline 7. ACTA PHARM JUGOSL (44) & 206.8 & 650 & 2032 & 312.6 & $1975-1991^{2}$ \\
\hline 8. FOOD TECHNOL BIOTECH (22) & 206.7 & 109 & 146 & 133.9 & $1996 \longrightarrow>^{3}$ \\
\hline 9. MATH INEQUAL APPL (31) & 154.6 & 56 & 40 & 71.4 & $1998 \longrightarrow$ \\
\hline 10. VET ARHIV (49) & 141.6 & 993 & 603 & 60.7 & $1975 \longrightarrow>^{4}$ \\
\hline 11. CHEM BIOCHEM ENG Q (13) & 128.2 & 306 & 437 & 142.8 & $1987 \longrightarrow$ \\
\hline 12. TRAVAUX ICSOBA (24) & 128.0 & 333 & 214 & 64.3 & $1975-1996$ \\
\hline 13. ACTA GEOL (26) & 100.1 & 69 & 79 & 114.5 & $1975 \longrightarrow$ \\
\hline 14. BILJESKE NOTES I OCE (7) & 95.1 & 39 & 36 & 92.3 & $1975 \longrightarrow$ \\
\hline 15. ACTA ENTOMOL JUGOSL (6) & 89.9 & 164 & 78 & 47.6 & $1975-1990$ \\
\hline 16. HVAR OBS B (52) & 85.6 & 227 & 402 & 177.1 & $1977 \longrightarrow$ \\
\hline 17. KRS JUGOSLAVIJE (27) & 77.3 & 15 & 14 & 93.3 & $1975-1991$ \\
\hline 18. KEM IND (18) & 72.3 & 1046 & 450 & 43.0 & $1975 \longrightarrow$ \\
\hline 19. CROATIAN MED J (35) & 63.6 & 497 & 510 & 102.6 & $1992 \longrightarrow>^{5}$ \\
\hline 20. STROJARSTVO (21) & 60.6 & 923 & 112 & 12.1 & $1975 \longrightarrow$ \\
\hline 21. IZV JUGOSL CENT KRIS (53) & 60.4 & 237 & 138 & 58.2 & $1975-1978^{6}$ \\
\hline 22. ACTA PARASITOL IUGOS (9) & 55.8 & 87 & 51 & 58.6 & $1975-1980$ \\
\hline 23. ARH HIG RADA TOKSIKO (46) & 52.6 & 995 & 595 & 59.8 & $1975 \longrightarrow$ \\
\hline 24. ACTA BOT CROAT (10) & 47.9 & 506 & 264 & 52.2 & $1975 \longrightarrow$ \\
\hline 25. CEMENT (15) & 47.6 & 107 & 13 & 12.2 & $1975-1988$ \\
\hline 26. ACTA PHARM (44) & 47.2 & 356 & 254 & 71.3 & $1992 \longrightarrow>^{7}$ \\
\hline 27. INT J ENG MODELLING (14) & 47.0 & 77 & 20 & 26.0 & $1991 \longrightarrow$ \\
\hline 28. KINESIOLOGY (36) & 45.4 & 60 & 16 & 26.7 & $1996 \longrightarrow>^{8}$ \\
\hline 29. METALURGIJA (24) & 45.3 & 536 & 122 & 22.8 & $1975 \longrightarrow$ \\
\hline 30. ACTA BIOL (5) & 42.2 & 54 & 41 & 75.9 & $1975 \longrightarrow$ \\
\hline 31. POLJOPRIVREDNA ZNANS (1) & 38.1 & 798 & 115 & 14.4 & $1975-1996^{9}$ \\
\hline 32. HIDROGRAFSKI GODISNJ (54) & 37.1 & 82 & 25 & 30.5 & $1975-1992$ \\
\hline 32. PREHRAM TEHNOL BIOT (22) & 36.7 & 727 & 173 & 23.8 & $1975-1995^{10}$ \\
\hline 34. GLASNIK SUMSKE POKUS (4) & 36.2 & 223 & 40 & 17.9 & $1975 \longrightarrow$ \\
\hline 35. FIZIKA B (55) & 33.6 & 154 & 109 & 70.8 & $1992 \longrightarrow>^{11}$ \\
\hline 36. TEKSTIL (50) & 32.9 & 2422 & 481 & 19.9 & $1975 \longrightarrow$ \\
\hline 37. GEOFIZIKA (27) & 32.1 & 124 & 48 & 38.7 & $1984 \longrightarrow$ \\
\hline 38. FIZIKA (55) & 31.4 & 1920 & 1271 & 66.2 & $1975-1991^{12}$ \\
\hline 39. FIZIKA A (55) & 30.7 & 198 & 128 & 64.7 & $1992 \longrightarrow>^{13}$ \\
\hline 40. PERIOD BIOL (5) & 29.4 & 4853 & 2568 & 52.9 & $1975 \longrightarrow$ \\
\hline 41. ACTA MED IUGOSL (35) & 28.4 & 640 & 293 & 45.8 & $1975-1991^{14}$ \\
\hline 42. ZBORNIK RADOVA FAKUL (21) & 27.7 & 362 & 20 & 5.5 & $1975 \longrightarrow$ \\
\hline 43. POLIMERI (12) & 26.9 & 489 & 122 & 24.9 & $1980 \longrightarrow$ \\
\hline 44. RASPRAVE PAPERS (28) & 26.3 & 109 & 45 & 41.3 & $1975-1990^{15}$ \\
\hline 45. GEOL CROAT (26) & 25.2 & 163 & 47 & 28.8 & $1992 \longrightarrow>^{16}$ \\
\hline 46. ACTA MED CROATICA (35) & 25.0 & 360 & 145 & 40.3 & $1991 \longrightarrow>^{17}$ \\
\hline 47. POLJOPRIVREDA (1) & 23.8 & 89 & 8 & 9.0 & $1995 \longrightarrow>^{18}$ \\
\hline 48. J COMPUT INFORM TECH (14) & 23.6 & 153 & 20 & 13.1 & $1993 \longrightarrow$ \\
\hline 49. KINEZIOLOGIJA (36) & 22.7 & 361 & 48 & 13.3 & $1975-1995^{19}$ \\
\hline 50. AUTOMATIKA (14) & 22.4 & 647 & 80 & 12.4 & $1975 \longrightarrow$ \\
\hline
\end{tabular}


Table 2. (cont.)

\begin{tabular}{|c|c|c|c|c|c|}
\hline $\begin{array}{l}\text { Journal title } \\
(\text { category from Table } 1)^{1}\end{array}$ & NCP & $\begin{array}{l}\text { Number of } \\
\text { papers }(P)\end{array}$ & $\begin{array}{l}\text { Number of } \\
\text { citations }(\mathrm{C})\end{array}$ & $100 \mathrm{C} / \mathrm{P}$ & $\begin{array}{c}\text { Publishing } \\
\text { period }\end{array}$ \\
\hline 51. JUGOSL GINEKOL PERIN (39) & 21.0 & 227 & 55 & 24.2 & $1985-1991^{20}$ \\
\hline 52. GOD JUGOSL CENT KRIS (53) & 20.3 & 724 & 142 & 19.6 & $1979-1990^{21}$ \\
\hline 53. LARUS (8) & 19.7 & 137 & 19 & 13.9 & $1975 \longrightarrow$ \\
\hline 54. PRAXIS VET (49) & 19.6 & 915 & 77 & 8.4 & $1975 \longrightarrow$ \\
\hline 55. HRVATSKI METEOROLOSK (28) & 18.3 & 66 & 19 & 28.8 & $1991-1997^{22}$ \\
\hline 56. GEOL VJESNIK (26) & 18.3 & 492 & 103 & 20.9 & $1975-1991^{23}$ \\
\hline 57. ACTA STOMATOL CROAT (32) & 16.5 & 977 & 116 & 11.8 & $1975 \longrightarrow$ \\
\hline 58. ACTA GEOGRAPHICA CRO (25) & 16.3 & 73 & 7 & 9.6 & $1992 \longrightarrow$ \\
\hline 59. ALCOHOLISM (48) & 16.0 & 291 & 56 & 19.2 & $1975 \longrightarrow$ \\
\hline 60. NEUROPSIHIJATRIJA (38) & 15.7 & 57 & 19 & 33.3 & $1975-1976^{24}$ \\
\hline 61. GEOGRAPHICAL PAPERS (25) & 14.9 & 91 & 8 & 8.8 & $1975-1991$ \\
\hline 62. B SCI A (51) & 14.7 & 92 & 57 & 62.0 & $1975-1976$ \\
\hline 63. MLJEKARSTVO (22) & 14.1 & 789 & 72 & 9.1 & $1975 \longrightarrow$ \\
\hline 64. ZNAN PRAK POLJOPR TE (1) & 13.6 & 506 & 26 & 5.1 & $1982-1994^{25}$ \\
\hline 65. KOSARKASKI MED VJESN (36) & 13.3 & 64 & 5 & 7.8 & $1986-1990^{26}$ \\
\hline 66. NAFTA ZAGREB (18) & 12.4 & 1256 & 93 & 7.4 & $1975 \longrightarrow$ \\
\hline 67. FARM GLAS (44) & 12.3 & 404 & 75 & 18.6 & $1975 \longrightarrow$ \\
\hline 68. PSYCHIAT DANUBINA (45) & 12.0 & 344 & 64 & 18.6 & $1989 \longrightarrow$ \\
\hline 69. JUGOSL GINEKOL OPSTE (39) & 11.4 & 494 & 65 & 13.1 & $1975-1984^{27}$ \\
\hline 70. GORIVA MAZIVA (18) & 11.3 & 459 & 31 & 6.8 & $1975 \longrightarrow$ \\
\hline 71. TEHNICKI VJESNIK (17) & 11.1 & 63 & 2 & 3.2 & $1994 \longrightarrow$ \\
\hline 72. VET STANICA (49) & 10.4 & 1006 & 45 & 4.5 & $1975 \longrightarrow$ \\
\hline 73. GEOGRAFSKI GLASNIK (25) & 10.2 & 283 & 17 & 6.0 & $1975 \longrightarrow$ \\
\hline 74. FRAGMENTA HERBOLOGIC (10) & 9.8 & 310 & 33 & 10.7 & $1976-1991^{28}$ \\
\hline 75. DIAB CROAT (33) & 9.1 & 976 & 201 & 20.6 & $1975 \longrightarrow$ \\
\hline 76. MATH COMMUN (30) & 8.9 & 69 & 2 & 2.9 & $1996 \longrightarrow$ \\
\hline 77. NATURA CROATICA (5) & 8.8 & 145 & 23 & 15.9 & $1992 \longrightarrow$ \\
\hline 78. LIJEC VJESN (35) & 8.6 & 2860 & 395 & 13.8 & $1975 \longrightarrow$ \\
\hline 79. ANN I FRANCAIS ZAGRE (51) & 8.3 & 63 & 22 & 34.9 & $1975-1987$ \\
\hline 80. KRMIVA (2) & 8.0 & 987 & 53 & 5.4 & $1975 \longrightarrow$ \\
\hline 81. STOCARSTVO (2) & 7.7 & 1073 & 55 & 5.1 & $1975 \longrightarrow$ \\
\hline 82. AGRONOMSKI GLASNIK (1) & 7.4 & 1040 & 29 & 2.8 & $1975 \longrightarrow$ \\
\hline 83. GEOGRAFSKI HORIZONT (25) & 7.2 & 285 & 12 & 4.2 & $1975-1997$ \\
\hline 84. RIBARSTVO (3) & 6.7 & 86 & 4 & 4.7 & $1992 \longrightarrow$ \\
\hline 85. ACTA BIOKOVICA (10) & 6.6 & 125 & 9 & 7.2 & 1981-1995 \\
\hline 86. S OTORHINOL ZAGREB (42) & 6.2 & 96 & 3 & 3.1 & 1992-1997 \\
\hline 87. RAD MED FAK ZAGREB (35) & 6.1 & 283 & 28 & 9.9 & $1975-1991^{29}$ \\
\hline 88. LJEVARSTVO (24) & 5.7 & 175 & 5 & 2.9 & $1975 \longrightarrow$ \\
\hline 89. RADOVI (4) & 5.7 & 389 & 11 & 2.8 & $1975 \longrightarrow$ \\
\hline 90. GYNAECOL PERINATOL (39) & 5.6 & 463 & 30 & 6.5 & $1992 \longrightarrow>^{30}$ \\
\hline 91. OPHTHALMOL CROAT (41) & 5.3 & 122 & 7 & 5.7 & $1992 \longrightarrow$ \\
\hline 92. SUMARSKI LIST (4) & 5.2 & 848 & 22 & 2.6 & $1975 \longrightarrow$ \\
\hline 93. JUG PEDIJAT (43) & 5.1 & 580 & 29 & 5.0 & $1975-1990$ \\
\hline 94. ENERGETICAR (16) & 4.8 & 342 & 7 & 2.0 & $1975-1991$ \\
\hline 95. CROAT J GASTROENTERO (34) & 4.5 & 159 & 13 & 8.2 & $1992 \longrightarrow$ \\
\hline 96. MORSKO RIBARSTVO (3) & 4.5 & 737 & 23 & 3.1 & $1975-1995$ \\
\hline 97. RIBARSTVO JUGOSLAVIJ (3) & 4.5 & 317 & 10 & 3.1 & 1975-1991 \\
\hline 98. EGE (16) & 4.5 & 674 & 13 & 1.9 & $1993 \longrightarrow$ \\
\hline 99. CROAT SPORTS MED J (36) & 4.4 & 117 & 3 & 2.6 & $1991 \longrightarrow$ \\
\hline 100. PHARMACA (44) & 4.4 & 407 & 27 & 6.6 & $1975 \longrightarrow$ \\
\hline
\end{tabular}


Table 2. (cont.)

\begin{tabular}{|c|c|c|c|c|c|}
\hline $\begin{array}{l}\text { Journal title } \\
(\text { category from Table } 1)^{1}\end{array}$ & NCP & $\begin{array}{l}\text { Number of } \\
\text { papers }(\mathrm{P})\end{array}$ & $\begin{array}{l}\text { Number of } \\
\text { citations (C) }\end{array}$ & $100 \mathrm{C} / \mathrm{P}$ & $\begin{array}{l}\text { Publishing } \\
\text { period }\end{array}$ \\
\hline 101. SUNCEVA ENERGIJA (16) & 4.2 & 166 & 3 & 1.8 & $1979-1994$ \\
\hline 102. S OTORHINOLARYNGOLOG (42) & 4.1 & 530 & 11 & 2.1 & $1975-1991$ \\
\hline 103. NEUROL CROATICA (38) & 4.0 & 905 & 78 & 8.6 & $1991 \longrightarrow>^{31}$ \\
\hline 104. BRODOGRADNJA (19) & 4.0 & 462 & 7 & 1.5 & $1975 \longrightarrow$ \\
\hline 105. ARHIV ZA ZASTITU MAJ (43) & 3.8 & 765 & 29 & 3.8 & $1975-1992^{32}$ \\
\hline 106. PAEDIAT CROAT (43) & 3.7 & 303 & 11 & 3.6 & $1993 \longrightarrow>^{33}$ \\
\hline 107. GLASNIK ZASTITE BILJ (1) & 3.6 & 806 & 11 & 1.4 & $1978 \longrightarrow>^{34}$ \\
\hline 108. ACTA FAC MED FLUM (35) & 3.5 & 299 & 17 & 5.7 & $1975 \longrightarrow$ \\
\hline 109. REUMATIZAM (47) & 3.5 & 707 & 44 & 6.2 & $1975 \longrightarrow$ \\
\hline 110. LIBRI ONCOL (40) & 3.4 & 802 & 60 & 7.5 & $1975 \longrightarrow$ \\
\hline 111. FRAGM PHYTOMHERBOL (10) & 3.2 & 58 & 2 & 3.5 & $1993 \longrightarrow>^{35}$ \\
\hline 112. POLJOPRIVREDNE AKTUA (1) & 3.1 & 1108 & 13 & 1.2 & $1975-1994$ \\
\hline 113. ZAVARIVANJE (24) & 3.0 & 587 & 9 & 1.5 & $1975 \longrightarrow$ \\
\hline 114. MED AN (37) & 3.0 & 285 & 16 & 5.6 & $1985-1993^{36}$ \\
\hline 115. AGR CONSPEC SCI (1) & 2.9 & 91 & 1 & 1.1 & $1997 \longrightarrow>^{37}$ \\
\hline 116. DRVNA IND (4) & 2.7 & 673 & 9 & 1.3 & $1975 \longrightarrow$ \\
\hline 117. ACTA GRAPH (17) & 2.7 & 130 & 1 & 0.8 & $1989 \longrightarrow$ \\
\hline 118. ELEKTROTEHNIKA (20) & 2.7 & 924 & 16 & 1.7 & $1975 \longrightarrow$ \\
\hline 119. GLASNIK HRVATSKOG DR (23) & 2.6 & 81 & 2 & 2.5 & $1991-1993^{38}$ \\
\hline 120. TROGLODYTES (8) & 2.5 & 111 & 2 & 1.8 & $1987-1995$ \\
\hline 121. MED JAD (37) & 2.3 & 854 & 37 & 4.3 & $1975 \longrightarrow$ \\
\hline 122. ACTA CLIN CROAT (37) & 2.2 & 343 & 14 & 4.1 & $1991 \longrightarrow>^{39}$ \\
\hline 123. NEUROLOGIJA (38) & 2.2 & 1215 & 56 & 4.6 & $1977-1990^{40}$ \\
\hline 124. GRADEVINAR (19) & 2.1 & 1237 & 10 & 0.8 & $1975 \longrightarrow$ \\
\hline 125. RUDARSKO GEOLOSKO NA (24) & 2.0 & 297 & 3 & 1.0 & $1989 \longrightarrow$ \\
\hline 126. ENERGIJA (16) & 2.0 & 918 & 8 & 0.9 & $1975 \longrightarrow$ \\
\hline 127. POMORSKI ZBORNIK (51) & 1.9 & 780 & 61 & 7.8 & $1975 \longrightarrow$ \\
\hline 128. KLIN MED (37) & 1.8 & 88 & 3 & 3.4 & $1992 \longrightarrow$ \\
\hline 129. SAOPCENJA (44) & 1.7 & 231 & 6 & 2.6 & $1975-1990$ \\
\hline 130. BILJNA ZAST (1) & 1.7 & 156 & 1 & 0.6 & $1975-1977^{41}$ \\
\hline 131. STRUKA ZNANOST (37) & 1.6 & 68 & 2 & 2.9 & $1980-1985$ \\
\hline 132. AN KLIN B M STOJANOV (37) & 1.4 & 482 & 13 & 2.7 & $1975-1990^{42}$ \\
\hline 133. PROMET ZAGREB (17) & 1.2 & 570 & 2 & 0.3 & $1989 \longrightarrow$ \\
\hline 134. MEDICINAR (35) & 1.2 & 305 & 6 & 2.0 & $1975 \longrightarrow$ \\
\hline 135. PRAXIS MED (37) & 1.1 & 464 & 10 & 2.2 & $1975-1990$ \\
\hline 136. KOZA OBUCA (17) & 1.1 & 1866 & 6 & 0.3 & $1975 \longrightarrow$ \\
\hline 137. MEDICINA (35) & 0.9 & 1235 & 17 & 1.4 & $1975 \longrightarrow$ \\
\hline 138. JADRANSKI ZBORNIK (51) & 0.4 & 272 & 5 & 1.8 & $1975-1997$ \\
\hline 139. ANALI OPCE BOLNICE S (37) & 0.4 & 283 & 2 & 0.7 & $1975-1984^{43}$ \\
\hline 140. ACTA MEDICOR (37) & 0.2 & 505 & 2 & 0.4 & $1975 \longrightarrow$ \\
\hline 141. ANAL OPCE BOLNIC J K (37) & 0.2 & 225 & 1 & 0.4 & $1975-1989$ \\
\hline 142. BIOCHEMIA MEDICA (23) & 0.0 & 318 & 0 & 0.0 & $1994 \longrightarrow>^{44}$ \\
\hline
\end{tabular}

${ }^{1}$ Journals included in WoS are underlined

${ }^{2}$ Changed to ACTA PHARM

${ }^{3}$ Changed from PREHRAM TEHNOL BIOT

${ }^{4}$ Indexed in WoS in part

${ }^{5}$ Changed from RAD MED FAK ZAGREB

${ }^{6}$ Changed to GOD JUGOSL CENT KRIS

${ }^{7}$ Changed from ACTA PHARM JUGOSL

${ }^{8}$ Changed from KINEZIOLOGIJA 


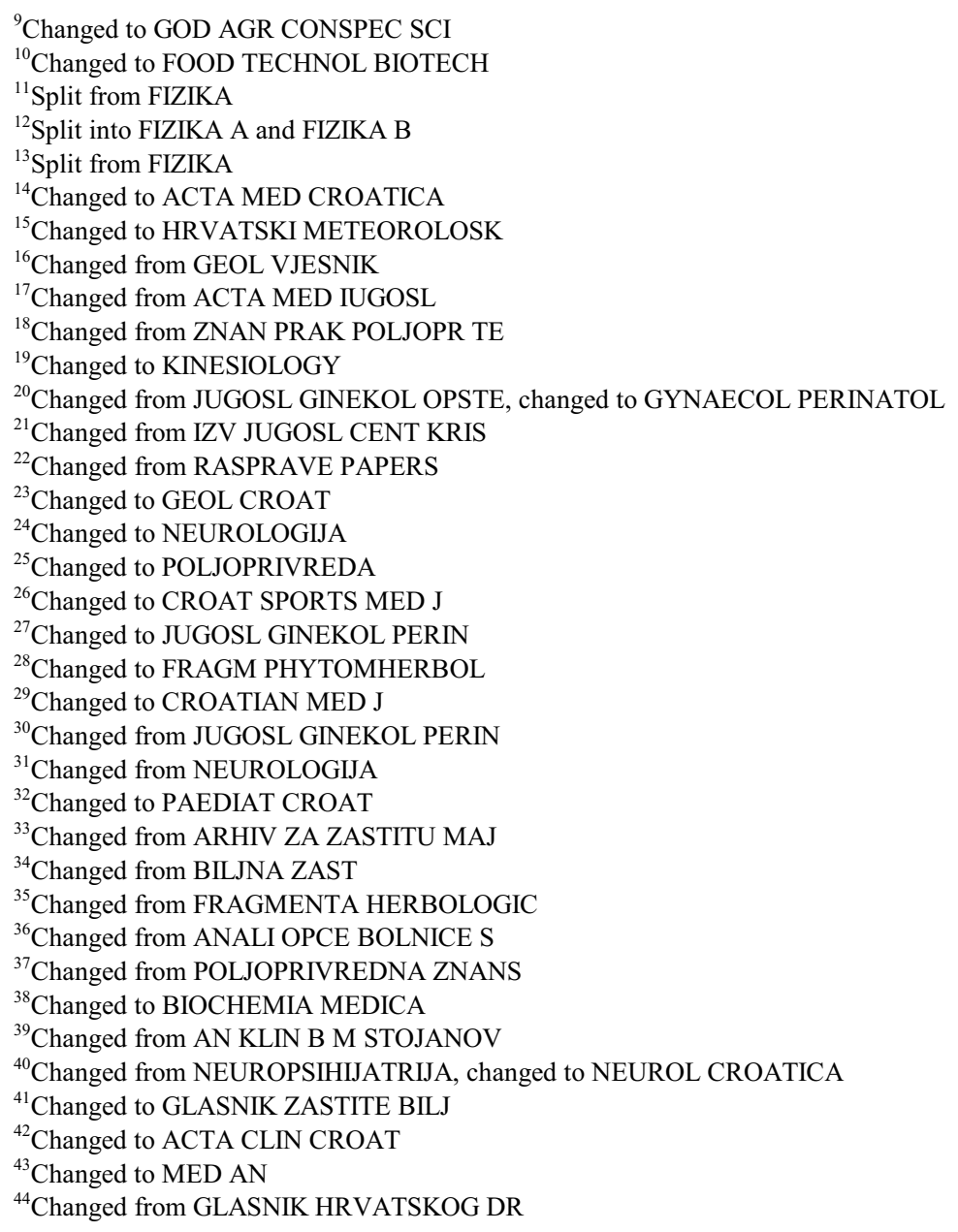

Biological sciences category in our sample is covered by the following fields: Biology, Entomology, Marine \& Freshwater Biology, Ornithology, Parasitology and Plant Sciences, and average value of $\mathrm{C} / \mathrm{P}=0.547$. Compared to ESI 1995-2005, this category has 10.58 citations per paper. A field with the highest $\mathrm{C} / \mathrm{P}=1.89$ is Marine \& Freshwater Biology, due to the journals Acta Adriatica and Bilješke Notes (Table 2). Both journals are published by Institute for Oceanography and Fisheries, Split. In addition the special value of these results lies in a fact that these journals are not indexed by SCI-expanded, which means that they have no self-citations. We could explain this pretty high SCI value of $\mathrm{C} / \mathrm{P}$ for these journals with the relevance of papers and also with an important fact that papers are published in English. 
$\mathrm{C} / \mathrm{P}$ value of Croatian journals for chemistry is 4.44 , which puts chemistry in the first place related to all other fields or categories. Compared to the ESI 1995-2005, an average value for this category is 7.95 citations per paper. However, real contribution appertain to journal Croat Chem Acta, as the most prestige Croatian journal with $\mathrm{C} / \mathrm{P}=5.57$ (see Table 2), indexed by SCI at the begging in 1974. In this case we could agree with SOMBATSOMPOP \& AL. [2002] that journals with older ages tended to have more opportunity to be cited.

Engineering is relatively broad category, represented in our sample with 12 fields. Depending on fields the oscillations in number of citations per paper is visible, e.g. Biotechnology \& Applied Microbiology with the highest C/P value, and Engineering and Medical Laboratory Technology with the lowest $\mathrm{C} / \mathrm{P}$. One of the factors contributing to this result in the field of Biotechnology \& Applied Microbiology is a journal Chem Biochem Eng $Q$ with $\mathrm{C} / \mathrm{P}=1.42$. This journal is indexed by SCIexpanded, and all papers are published in English. Also, it is important to mention the fact that we have only one journal in this field. Comparing our result for Engineering as a category, C/P $=0.126$, with ESI 1995-2005, number of citations per paper is 3.08. One of the reasons for a pretty low value of citations per paper for Croatian journals in this category could be the language barrier. Namely, the majority of journals publish articles only in Croatian.

Geosciences are ranged in the fourth place, in consideration to the average number of citations per paper $(\mathrm{C} / \mathrm{P}=0.253)$ comparing to the ESI 1995-2005 data (7.49). Two extremes are noticeable: Geography $(\mathrm{C} / \mathrm{P}=0.07)$ and Palaeontology $(\mathrm{C} / \mathrm{P}=1.96)$. It is important to stress out that Palaeontology as a field in our sample is represented with only one journal, with relatively low number of papers and which ceased at the beginning of nineties (see Table 2). Average paper received almost 2 citations (1.96). In the filed of Geography we have four journals and all of the papers are published in Croatian.

Data obtained for Mathematics show high homogeneity of the field. Included journals have an average $\mathrm{C} / \mathrm{P}=0.719$, while the ESI 1995-2005 data for this field reports about 2.60 citations per paper. Comparing these two results it is clear that papers published in Croatian mathematical journals received approximately one third of the world average citations for this field.

Similar to the results obtained for Engineering, in the category of Medical Sciences differences in an average number of citations per papers for some fields are noticeable. Pharmacology \& Pharmacy with $\mathrm{C} / \mathrm{P}=1.16$ is a field with the best results, owing to the journal Acta Pharm Jugosl. This journal published papers in English and it was indexed by SCI until 1991. According to the ESI 1995-2005 data for the field of Farmacology \& Toxicology value of the mean citations per paper was 9.24. The field with relatively high mean citations per paper in this category is Public, Environmental \& Occupational Health, especially thanks to the journal Arh Hig Rada Toksiko, with C/P $=0.598$. 
Physics is a category, after chemistry, with the highest number of citations per paper. In this sample it is represented with four fields. Oceanography with $\mathrm{C} / \mathrm{P}=3.30$, is a field with the highest mean citations per paper, owing to contribution of the journal Thalassia Jugoslavica. This journal is ranked in the first place by mean number of citations per paper $(\mathrm{C} / \mathrm{P}=3.99)$. Physics as a category, according to the ESI 1995-2005 data has a mean of 7.12 citations per paper. Comparing the data obtained for the journal Thalassia Jugoslavica with the ESI 1995-2005 data, it is noticeable that this journal received more than a half of the world average number of citations per paper. Additionally, it is important to stress out that this journal has never been indexed by SCI database. Therefore, all received citations are free of journal self-citations, and their recognitions are a product of relevant papers. Also, it is worth to mention that all of papers were published in English.

The second ranked field in this category is Astronomy and Astrophysics, represented by the journal Hvar Obs B, with $\mathrm{C} / \mathrm{P}=1.77$. Neither this journal was indexed by SCI, but all papers were published in English.

While we do not have an indicator like IF, for the purpose of this research we must introduce a new indicator, NCP (Normalized citations per paper divided by IF of categories for year 1989, as a mean year between 1975-2001). A status for each journal could be more objective if we take a mean year of journal publishing period instead of the year 1989 .

The main reason for this decision lies is in the fact of huge time consuming job, and as we mentioned earlier, IF of some fields does not change significantly during the time period.

Our results show that among 11 journals with $\mathrm{C} / \mathrm{P}>1.00$, two journals, Hvar Obs $B$ and Croatian Med J, with calculated NCP, gained lower status. Their NCP value is less than 100 (see Table 2) due to relatively high value of category IF.

Four journals have a $\mathrm{C} / \mathrm{P}$ less than $1(0.61<\mathrm{C} / \mathrm{P}<0.77)$, but their better NCP rank is a consequence of a relatively low IF of their categories. Those journals are: Travaux ICSOBA, Vet Arhiv, Mathematical Inequalities and Applications and Glasnik Mat. Travaux ICSOBA has ceased, and it has never been indexed by SCI-expanded. Journal Glasnik Mat, even though never indexed by SCI is ranked by its NCP in the fifth place on the scale of 142 Croatian journals. Vet Arhiv was irregularly indexed by SCIexpanded. Only one journal from this group, Mathematical Inequalities and Applications, is indexed by SCI-expanded since the first volume.

It was mentioned before, and it is visible in Table 2, that 10 journals from our sample are indexed by SCI-expanded (underlined titles). Potentially, those journals have a higher number of citations per paper due to the possibility of journal self-citations. Those journals are not in the same position compared to the remaining 132 journals, without an option of journal self-citations. To solve this problem two methodological approaches can be used: to exclude journal self-citations for SCI indexed journals, or to 
add journal self-citations to all of the journals. For example, the calculation of impact factor for journal Kem Ind [ANDREIS, 1998] shows that the number of journal selfcitations in the 1975-1999 period is 498. With an addition of these citations the total number of SCI-expanded citations per 100 papers (450) will be more than $110 \%$ higher, and this journal can be compared with the journal Mathematical Inequalities and Applications (number 9 in Table 2)

Among the journals with the highest impact measured by NCP (in the range from 486.2 to 100.1) which have never been indexed by SCI-expanded, and have ceased in the meantime, are Thalassia Jugoslavica, Paleontologia Jugoslavica, Travaux ICSOBA, and Acta Geologica. Journal Acta Pharm Jugosl was indexed by SCI until 1991, coincidently with the change of the name. According to the NCP value this journal is ranked as $7^{\text {th }}$, but with the name changed to Acta Pharmac, it decreased to $26^{\text {th }}$. We suppose that one of the reasons for this decrease could be exclusion of journal selfcitation. Namely, CAMPANARIO \& GONZALEZ [2006] in their study identified that large number of journal self-citations contributed to the impact factor.

Among $13(9.1 \%)$ journals with $\mathrm{NCP}>100.5$ journals were, and 4 of them are currently indexed by SCI-expanded (Table 2). Remaining 4 journals from this group, which are active (Ann Forestales, Glasnik Mat, Acta Adriatica and Vet Arhiv), could be covered by SCI-expanded.

However, it should be mentioned that some journals contain meeting abstracts, which were partly indexed by SCI-expanded as source items in the time span 1975-1989. Some issues of certain volume of Period Biol, particularly supplements containing only meeting abstracts, as well as extended abstracts that are sometimes classified as articles. In our study all type of papers are taken as source items, regardless on article type (review, article, note, letter, meeting abstract). Since some journals have a large number of meeting abstracts whose number of citations is generally very low, in Table 3 are presented $100 \mathrm{C} / \mathrm{P}$ according to the type of article. Generally, meeting abstracts are poorly cited, as show the data for SCI-expanded indexed journal Period Biol: 2136 articles have $100 \mathrm{C} / \mathrm{P}=100.7$, while 2717 meeting abstracts $(56 \%$ of source items) have $100 \mathrm{C} / \mathrm{P}=10.1$ resulting in a total $100 \mathrm{C} / \mathrm{P}=52.9$ for 4853 source items.

An exception is Izv Jugosl Cent Kris whose 70 meeting abstracts (29.5\% of source items) are significantly higher cited $(100 \mathrm{C} / \mathrm{P}=88.6)$ compared to articles $(100 \mathrm{C} / \mathrm{P}=45.5)$. The reason for relatively large number of citations related to meeting abstracts is the specific nature of abstracts in the field of crystallography: crystallographic data presented in few sentences are sometimes a valuable reference for scientists in this field.

An additional parameter strongly related to IF is the uncitedness. VAN LEEUWEN \& MOED [2005] analysed the correlation between uncitedness and IF for 1995 and have shown that journals containing a smaller number of publications tend to have a larger share in uncited papers, as well as the fact that IF 1 is generally correlated with uncitedness of $50 \%$. However, our data for journals having citations per paper (CP) $>1$, 
presented in Table 4, show that the number of papers is not generally directly correlated to the uncitidness, as well as the uncitedness does not have a major influence on CP. For example, the same number of citations arises from three papers with 50 citations or from 150 papers with one citation that will significantly decrease uncitedness.

Journals with highest $\mathrm{CP}$ have a dominant contribution from highly cited papers (first three journals). Although journals No. 5 and 6 have a similar CP (about 1.95) they significantly differ in uncitedness $(34.5 \%$ and $65.5 \%$, respectively) because of the different contribution of papers with less than 10 or more than 10 citations. However, data for all of 142 journals (Figure 1) show that the correlation between CP and uncitedness does exists for journals with less than one citation per paper.

The main reason for very poor citations of more than 90\% STM Croatian journals could be the same as stressed out by REN \& ROUSSEAU [2002] for Chinese and Japanese journals: The influence of scientific evaluation procedures, and local versus international science, language problems, and ZITT \& AL. [2003]: ...the lower impact of the nationally-oriented journals is associated with a low internationalization of journals.

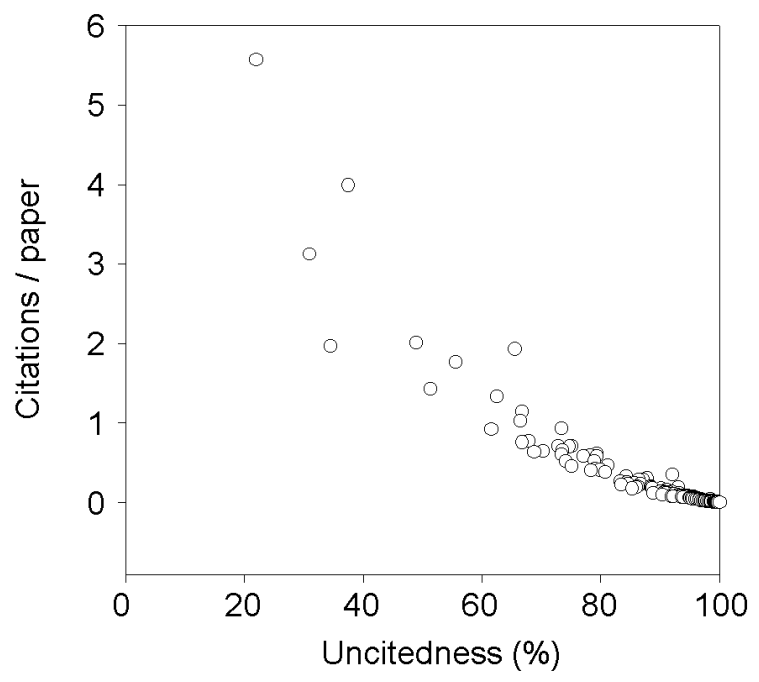

Figure 1. Distribution of citations per paper and uncitedness for Croatian journals 
M. ANDREIS \& M. JOKIĆ: An impact of Croatian journals measured by citation analysis

Table 3. Contribution of meeting abstracts (MA) to the number of citations

\begin{tabular}{lccrrc}
\hline Journal & Source items & $100 \mathrm{C} / \mathrm{P}$ & $\mathrm{MA}$ & $100 \mathrm{C} / \mathrm{P}$ & $\% \mathrm{MA}$ \\
\hline GOD JUGOSL CENT KRIST & 724 & 19.6 & 664 & 6.3 & 91.7 \\
NEUROLOGIJA & 1215 & 4.6 & 876 & 0.1 & 72.1 \\
PERIOD BIOL & 4853 & 52.9 & 2717 & 10.1 & 56.0 \\
REUMATIZAM & 707 & 6.2 & 264 & 2.6 & 37.3 \\
IZV JUGOSL CENT KRIST & 237 & 58.2 & 70 & 88.6 & 29.5 \\
PREHRAM TEHNOL BIOT & 727 & 23.8 & 213 & 0.5 & 29.3 \\
THALASSIA JUGOSL & 356 & 399.2 & 44 & 90.9 & 12.3 \\
\hline
\end{tabular}

Table 4. Uncitedness and distribution of citations for journals with NCP $>100$

\begin{tabular}{|c|c|c|c|c|c|c|c|}
\hline \multirow[t]{2}{*}{ Journal title } & \multirow[t]{2}{*}{$\mathrm{NCP}$} & \multirow[t]{2}{*}{ Papers } & \multicolumn{5}{|c|}{ Distribution of citations (\%) } \\
\hline & & & 0 & 1 & $2-9$ & $10-49$ & $>50$ \\
\hline 1. THALASSIA JUGOSL & 486.2 & 356 & 37.3 & 14.9 & 35.4 & 11.8 & 0.6 \\
\hline 2. CROAT CHEM ACTA & 410.1 & 1825 & 21.9 & 15.6 & 46.0 & 15.8 & 0.7 \\
\hline 3. $\overline{\text { ANN FORESTALES }}$ & 390.1 & 58 & 65.5 & 13.8 & 15.5 & 5.1 & - \\
\hline 4. PALAEONTOLOGIA JUGOS & 289.0 & 29 & 34.5 & 27.6 & 34.5 & 3.5 & - \\
\hline 5. GLASNIK MAT & 237.3 & 875 & 67.8 & 14.8 & 16.5 & 0.8 & - \\
\hline 6. ACTA ADRIATICA & 207.2 & 327 & 48.9 & 14.7 & 33.0 & 3.3 & - \\
\hline 7. ACTA PHARM JUGOSL & 206.8 & 650 & 30.9 & 20.5 & 42.8 & 5.5 & 0.3 \\
\hline 8. FOOD TECHNOL BIOTECH & 206.7 & 109 & 62.4 & 9.1 & 27.5 & 1.8 & - \\
\hline 9. $\overline{\text { MATH INEQUAL APPL }}$ & 154.6 & 56 & 75.0 & 10.7 & 12.5 & 1.8 & - \\
\hline 10. VET ARHIV & 141.6 & 993 & 73.3 & 15.1 & 10.9 & 0.6 & - \\
\hline 11. CHEM BIOCHEM ENG Q & 128.2 & 306 & 51.3 & 19.1 & 27.1 & 2.0 & - \\
\hline 12. TRAVAUX ICSOBA & 128.0 & 333 & 68.7 & 18.0 & 12.9 & 0.3 & - \\
\hline 13. ACTA GEOL & 100.1 & 69 & 66.7 & 10.1 & 20.3 & 2.9 & - \\
\hline
\end{tabular}

Under the pressure of scientific evaluation procedures Croatian scientists also tend to publish their more important works in international journals. The reasons for publishing articles in Croatian journals could be of local importance, rejections by international journals or issues of national science policy.

As our results showed, publishing papers only in Croatian definitely is a barrier for the international recognition. Namely, all of the journals with $\mathrm{NCP}>100$ and $\mathrm{CP}>1$, publish their papers in English.

\section{Type of errors detected by citation analysis}

A classification of the type of errors in the process of citation is explained in detail by GLÄNZEL \& AL. [2003]. These authors distinguish four main sources of errors: errors caused by citing authors; errors caused by the editors of the journals; errors caused by the database producer; and errors caused by the user of the bibliographic database. The authors themselves are probably responsible for most of the errors that have an effect on citation analyses. A paper that received 138 citations, had 25 incorrect citations. The error caused by citing authors amounts to $18.1 \%$. 
Although our search of SCI-expanded by both, journal title and author name, resulted in 10772 items (raw data), only 9007 of those papers do really exist, i.e. about 1700 items can not be identified at all. Raw database contains at least $17 \%$ of "ghost" articles that do not exist. Our sample, after the correction contained 28397 citations of 10772 items, which is about 0.4 citations per paper or about 2.6 citations per cited paper.

The raw and corrected data of cited papers from Croatian journals is presented in Table 5 .

Table 5. Distribution of citations for raw and corrected data

\begin{tabular}{lllllllll}
\hline Number of citations & 1 & 2 & 3 & 4 & $5-9$ & $10-24$ & $25-49$ & $>50$ \\
\hline Raw data (10772) & 6409 & 1765 & 790 & 499 & 865 & 367 & 63 & 14 \\
Corrected data (9007) & 4513 & 1701 & 811 & 520 & 953 & 420 & 73 & 16 \\
Difference & 1896 & 64 & -21 & -21 & -88 & -53 & -10 & -2 \\
\hline
\end{tabular}

The major contribution to the distribution of citations originates from a single incorrectly cited article (2307 items). They are also responsible for the fact that papers being cited three or more times after the correction, have more citations compared to the raw data.

Since 3717 (about 34\%) cited items contain at least one error, the article identification is made impossible. A detailed information on the accuracy of SCIexpanded data is obtained by analysis of errors in 10772 cited items from Croatian journals and the results show that more than $1 / 3$ of items can not be identified (Table 6).

About 18\% of articles (1627) originate from Croat Chem Acta (SCI-expanded indexed journal) with article-to-article link of about 91\% (1485 articles). All other journals including Period Biol and Acta Pharm Jugos (partly indexed by SCI-expanded) have an article-to-article link of about $73 \%$. Although $2563(69 \%)$ of incorrectly cited items contain only one out of six possible errors, the most frequent individual error ( $37 \%$ with respect to items) as well as general (26\% with respect to all errors) is an incorrect journal title. That indicates that analysis of Croatian journals can not be made by searching by journal title only.

However, for the purpose of IF, only two parameters in cited items are important: journal's title and year of publication (numerous misspelled first author's name/initials, journal's volume or paper's page have no effect on IF). The most important parameter for journal analysis is the title. Journals as a SCI-expanded source items generally have a unique abbreviation (limited to 11 characters in SCI or 21 characters in SCIexpanded), while source items not covered by SCI-expanded are abbreviated nonuniformly, according to the references in citing paper, and in most cases can not be identified at all. One of the numerous examples of the variations of cited articles in Croatian journals is shown in Table 7. 
M. ANDREIS \& M. JOKIĆ: An impact of Croatian journals measured by citation analysis

Table 6. Type of errors in raw data: 3717 (34.5\%) cited items containing at least one error that article-to-article-link makes impossible

\begin{tabular}{|c|c|c|c|c|c|c|}
\hline \multirow{2}{*}{$\begin{array}{c}\text { Number of items } \\
\text { [Numbers of errors } \\
\text { in item] }\end{array}$} & \multicolumn{6}{|c|}{ Type of errors } \\
\hline & Name & Initial & Journal & Volume & Page & Year \\
\hline $843[1]$ & - & - & 843 & - & - & - \\
\hline $596[1]$ & - & - & - & - & 596 & - \\
\hline $445[1]$ & - & - & - & 445 & - & - \\
\hline $262[1]$ & 262 & - & - & - & - & - \\
\hline $258[1]$ & - & - & - & - & - & 258 \\
\hline $226[2]$ & 226 & 226 & - & - & - & - \\
\hline $159[1]$ & - & 159 & - & - & - & - \\
\hline $148[2]$ & - & - & 148 & - & 148 & - \\
\hline $143[2]$ & - & - & - & 143 & 143 & - \\
\hline $103[2]$ & - & - & 103 & 103 & - & - \\
\hline $62[3]$ & - & - & - & 62 & 62 & 62 \\
\hline $56[2]$ & 56 & - & 56 & - & - & - \\
\hline $49[3]$ & - & - & 49 & 49 & 49 & - \\
\hline $41[3]$ & 41 & & 41 & 41 & - & - \\
\hline 39 [2] & - & - & 39 & - & - & 39 \\
\hline $29[2]$ & - & 29 & 29 & - & - & - \\
\hline 29 [2] & - & - & - & - & 29 & 29 \\
\hline $27[3]$ & 27 & 27 & - & - & 27 & - \\
\hline $22[2]$ & - & 22 & - & - & 22 & - \\
\hline $18[2]$ & - & 18 & - & 18 & - & - \\
\hline 18 [2] & 18 & - & - & - & 18 & - \\
\hline $16[2]$ & - & - & - & 16 & - & 16 \\
\hline $15[2]$ & 15 & - & - & 15 & - & - \\
\hline 14 [4] & - & - & 14 & 14 & 14 & 14 \\
\hline $10[3]$ & 10 & 10 & - & - & - & 10 \\
\hline $9[3]$ & 9 & 9 & - & 9 & - & - \\
\hline 7 [2] & 7 & - & - & - & - & 7 \\
\hline $7[4]$ & 7 & 7 & 7 & - & 7 & - \\
\hline 6 [3] & - & 6 & 6 & - & 6 & - \\
\hline $6[3]$ & 6 & - & 6 & - & 6 & - \\
\hline 6 [3] & 6 & - & 6 & 6 & - & - \\
\hline $6[4]$ & 6 & 6 & - & 6 & 6 & - \\
\hline 5 [2] & - & 5 & - & - & - & 5 \\
\hline 5 [3] & - & 5 & 5 & 5 & - & - \\
\hline $4[3]$ & - & - & 4 & - & 4 & 4 \\
\hline 3 [3] & - & - & 3 & 3 & - & 3 \\
\hline 3 [3] & 3 & - & 3 & - & - & 3 \\
\hline $3[4]$ & - & 3 & - & 3 & 3 & 3 \\
\hline $2[3]$ & - & 2 & 2 & - & - & 2 \\
\hline $2[3]$ & 2 & - & - & 2 & 2 & - \\
\hline $2[4]$ & 2 & - & - & 2 & 2 & 2 \\
\hline $2[4]$ & 2 & 2 & 2 & 2 & - & - \\
\hline $2[4]$ & - & 2 & 2 & 2 & 2 & - \\
\hline $1[3]$ & - & 1 & - & 1 & - & 1 \\
\hline $1[3]$ & - & 1 & - & - & 1 & 1 \\
\hline 1 [3] & - & 1 & - & 1 & 1 & - \\
\hline $1[4]$ & - & 1 & 1 & - & 1 & 1 \\
\hline $1[4]$ & 1 & - & 1 & 1 & 1 & - \\
\hline $1[4]$ & 1 & 1 & - & - & 1 & 1 \\
\hline $1[4]$ & 1 & 1 & - & 1 & - & 1 \\
\hline $1[5]$ & - & 1 & 1 & 1 & 1 & 1 \\
\hline $1[6]$ & 1 & 1 & 1 & 1 & 1 & 1 \\
\hline 3717 & 709 & 587 & 1372 & 911 & 1153 & 464 \\
\hline
\end{tabular}


M. ANDREIS \& M. JOKIĆ: An impact of Croatian journals measured by citation analysis

Table 7. An example of the paper and journal identification in SCI-expanded database

\begin{tabular}{|c|c|c|c|c|c|c|}
\hline Cited author & Cited journal & Vol. & Page & Year & \multicolumn{2}{|c|}{ Citations } \\
\hline & & & & & raw & corrected \\
\hline DIRIENZO F & 2ND YUG IT CRYST C D & & A102 & 1976 & 9 & - \\
\hline DIRIENZO F & CRYSTALLOGR C DUBROV & & A102 & 1976 & 1 & - \\
\hline DIRIENZO A & P YUGOSL CENT CRYS S & 11 & A102 & 1976 & 4 & - \\
\hline RIENZO F & P YUGOSLAV CTR CRY S & 11 & $\mathrm{~A} 102$ & 1976 & 1 & - \\
\hline DIRIENZO F & P YUGOSL CENT CRYS S & 11 & A102 & 1976 & 2 & - \\
\hline RIENZO FD & IZJV JUGOSLAV CTR S & 11 & A102 & 1976 & 1 & - \\
\hline DIRIENZO A & P YUGOSLV CENT C A S & 102 & & 1976 & 1 & - \\
\hline DIRIENZO F & 2ND YUG IT CRYST C D & & & 1976 & 1 & - \\
\hline DIRIENZO F* & IZV JUGOSL CENT KRIS & 11 & $\mathbf{A 1 0 2}$ & 1976 & - & 20 \\
\hline
\end{tabular}

*Correct citation of the article that can not be found in SCI-expanded

The full journal name (Izvještaj jugoslavenskog centra za kristalografiju) is most frequently abbreviated as Izv Jugosl Cent Kris. However, none of the shown cited items have this, or similar, abbreviation (Izjv instead of $I z v$ or $I z v j$ is a typing error), while English version ( $P$ Yugoslav Ctr Cryst) and its variations are not an appropriate journal abbreviation. The data show that no citations can be found using the search by journal title only, while four out of eight cited items can not be found using the search by authors name and initial.

LANGE [2002] described confusion regarding the abbreviations of two journal titles (Educational Research and Educational Researcher), which led to Educational Researcher being ascribed an exaggerated impact for a period of almost 20 years.

Searching for citations of Croatian authors and journals shows that cited English version of a non-English journal titles contributes to citations of well-known journals, for example J Phys Chem instead of Z Phys Chem or Chem Ind instead of Croatian Kem Ind). If the cited article is published a year or two before the citing article, it can influence the IF of a well-known journal. Even when the journal name is a single sixcharacter word such as Fizika (i.e. physics) only about $53 \%$ of items, or about $59 \%$ of citations can be found by searching the journal title (Table 8).

The fact that this journal has a relatively simple title and can be found with 40 different abbreviations, supports the conclusion that most of the Croatian journals can not be identified according to the abbreviated titles of cited works in SCI-expanded database. In addition, this example points out the unsolved problem of correct citing of supplements in the SCI-expanded system. Citations like Fizika S2 or Fizika S1 are related to supplements of certain volumes, and "S2" and "S1" are not a part of journal's title, as listed in SCI-expanded. An additional contribution to the IF can be the fact that two or more journals have the same journal title, or that a book of the same title also exists. For example, besides 313 items correctly cited as Fizika, there are also 104 items titled Fizika that are related either to a Russian journal or to Croatian or Russian books. 
M. ANDREIS \& M. JOKIĆ: An impact of Croatian journals measured by citation analysis

Table 8. An example of differences in citing journal Fizika

\begin{tabular}{|c|c|c|c|c|c|}
\hline \multirow[b]{2}{*}{ Cited journal title } & & \multicolumn{2}{|c|}{ Items } & \multicolumn{2}{|c|}{ Citations } \\
\hline & & number & $\%$ & number & $\%$ \\
\hline FIZIKA & & 313 & 53.3 & 757 & 59.5 \\
\hline FIZIKA ZAGREB & & 41 & 7.0 & 99 & 7.8 \\
\hline FIZIKA S2 & & 32 & 5.5 & 79 & 6.2 \\
\hline FIZIKA S1 & & 29 & 4.9 & 40 & 3.1 \\
\hline FIZIKA S & & 29 & 4.9 & 35 & 2.8 \\
\hline FIZIKA & $\mathrm{S} 2$ & 27 & 4.6 & 32 & 2.5 \\
\hline FIZIKA S3 & & 20 & 3.4 & 56 & 4.4 \\
\hline FIZIKA & S & 14 & 2.4 & 20 & 1.6 \\
\hline FISIKA & & 6 & 1.0 & 15 & 1.2 \\
\hline FIZ & & 6 & 1.0 & 13 & 1.0 \\
\hline FIZIKA & $\mathrm{S} 1$ & 6 & 1.0 & 6 & 0.5 \\
\hline FIZIKA & S3 & 5 & 0.9 & 16 & 1.3 \\
\hline FIZIKA ZAGREB & S & 5 & 0.9 & 8 & 0.6 \\
\hline FIZIKA ZAGREB S2 & & 4 & 0.7 & 6 & 0.5 \\
\hline FIZIKA ZAGREB S3 & & 4 & 0.7 & 17 & 1.3 \\
\hline FIZ S & & 4 & 0.7 & 8 & 0.6 \\
\hline FIZIKA ZAGREB S & & 3 & 0.5 & 3 & 0.2 \\
\hline FIZIKA ZAGREB SI & & 3 & 0.5 & 3 & 0.2 \\
\hline FIZIKA YUGOSLAVIA & & 3 & 0.5 & 9 & 0.7 \\
\hline PHYSICA S3 & & 3 & 0.5 & 3 & 0.2 \\
\hline FIZICA S2 & & 2 & 0.3 & 4 & 0.3 \\
\hline FIZICA & & 2 & 0.3 & 2 & 0.2 \\
\hline FIZ ZAGREB & & 2 & 0.3 & 6 & 0.5 \\
\hline FIZ YU & & 2 & 0.3 & 7 & 0.6 \\
\hline FIZIKA ZAGREB & $\mathrm{S} 2$ & 2 & 0.3 & 3 & 0.2 \\
\hline FIZIKA YUGOSLAVIA & S & 2 & 0.3 & 2 & 0.2 \\
\hline FIZ SI & & 1 & 0.2 & 2 & 0.2 \\
\hline FIZIKA A & & 1 & 0.2 & 1 & 0.1 \\
\hline FIZIKA YUGOSLAVIA & S & 1 & 0.2 & 1 & 0.1 \\
\hline FIZ & $\mathrm{S} 2$ & 1 & 0.2 & 3 & 0.2 \\
\hline FISIKA & S & 1 & 0.2 & 1 & 0.1 \\
\hline FIZ S3 & & 1 & 0.2 & 1 & 0.1 \\
\hline FIZIKA ZAGREB & $\mathrm{S} 1$ & 1 & 0.2 & 1 & 0.1 \\
\hline FIZIKA YUGOSLAVIA & $\mathrm{S} 2$ & 1 & 0.2 & 2 & 0.2 \\
\hline FISIKA S3 & & 1 & 0.2 & 1 & 0.1 \\
\hline FIZ YUGOSLAVIA & & 1 & 0.2 & 1 & 0.1 \\
\hline FISIKA S2 & & 1 & 0.2 & 1 & 0.1 \\
\hline FIZIKA SFRJ & & 1 & 0.2 & 2 & 0.2 \\
\hline FIZXIKA & & 1 & 0.2 & 1 & 0.1 \\
\hline FYSIKA & & 1 & 0.2 & 1 & 0.1 \\
\hline FYZIKA & & 1 & 0.2 & 1 & 0.1 \\
\hline HVAR FIZIKA YUGOSI & LAV & 1 & 0.2 & 1 & 0.1 \\
\hline FIZIKA YUG & & 1 & 0.2 & 1 & 0.1 \\
\hline PHYSIKA ZAGREB & & 1 & 0.2 & 1 & 0.1 \\
\hline
\end{tabular}

SCI-expanded database unifies titles only for indexed journals, while all other source items are abbreviated on the basis of the title given in the citing article. Consequently, numerous variations of journal titles can be found for the same journal. Some of them are considered as alternate titles (such as Chem Biochem Eng $Q$ or Chem Bioch Eng Q) while some items contain errors that make journal's identification impossible or relate cited title to some other journal (such as Chem Bioch $Q$ or Chem Bioeng $Q$ ). 


\section{Conclusion}

The aim of this research was to obtain an insight into the potential significance of papers published in Croatian journals which deal with the STM for the time span 1975-1998, by measuring their citedness within SCI-expanded database for 1975-2001. Among 142 journals classified in 10 categories and 55 fields respectively, according to JCR distribution, 10 journals were indexed by SCI-expanded database. Rest of the journals, more than $90 \%$, have never been indexed by ISI, but they did have a chance to be cited either by Croatian authors who published papers in journals indexed by SCI-expanded or by foreign authors.

For the purpose of this research we introduced a new scientometric indicator Normalized number of Citations per 100 Papers (NCP) that allows us direct comparison of the journals from various categories $\left(\mathrm{NCP}=100 \mathrm{C} / \mathrm{P} / \mathrm{IF}_{1989}\right)$. The year 1989 was chosen as a mean value for time span 1975-2001.

Only 11 journals from our sample $(7.7 \%)$ got an average of one or more citations per paper. In that group, in the time span studied, only 5 were indexed by SCIexpanded. Results show that $48.6 \%$ of Croatian journals have $\mathrm{NCP}<10.0$, and $53.5 \%$ journals have $\mathrm{CP}<0.1$. A more objective indicator concerning the field of subject is definitely NCP.

By citation analysis of journals, correction of incorrectly cited articles has been made and it has been established that $69 \%$ of papers contains at least one out of six identification parameters used to link citation to the original paper. The most frequent error, both individual (37\% with respect to items) and general (26\% with respect to all errors), is the incorrect journal title that has a significant influence on recognition of Croatian journals.

Obtained results arise a question of the importance of Croatian journals, and a necessity of funding their publishing. Journals covering the STM should basically have no boundaries from scientific point of view. Justification for the publishing these journals could be found in the research conducted by SANZ \& AL. [1995]. They established that the same group of scientists publishes the applied research results in national journals while basic research results are being published in international journals.

Results of this research could be usefull for journal editors as a guideline for adapting their editorial policy in order to improve a reception of their journals on an international level.

This article is dedicated to the memory of our friend and colleague Dr. Branimir Klaić, who left us too early.

This work was supported by the Croatian Ministry of Science, Education and Sport grants Nos. 098-0982915-2939 and Nos. 132-0000000-1489. 


\section{References}

ANDREIS, M. (1998), Primjena scientometrije u vrednovanju domaćih časopisa i znanstvene produkcije prema bazama ISI: kritička analiza. In: D. POLŠEK (Ed.), Vidljiva i nevidljiva akademija, Institut društvenih znanosti Ivo Pilar, Zagreb, pp.111-119.

Araujo Ruiz, J. A., Van Hooydonk, G., Torricella Morales, R. G., Arencibia Jorge, R. (2005), Cuban scientific articles in ISI Citation Indexes and Cuba Ciencias databases (1988-2003), Scientometrics, $65: 161-171$.

BRown, L. D. (2003), Ranking journals using social science research network downloads, Review of Quantitative Finance and Accounting, $20: 291-307$.

Buela-Casal, G., Perakakis P., Taylor, M., Checa, P. (2006), Measuring internationality: Reflections and perspectives on academic journals, Scientometrics, 67 : 45-65.

Campanario, J. M., Gonzalez, L. (2006), Journal self-citations that contribute to the impact factor: documents labelled "editorial material" in journals covered by Science Citation Index. Scientometrics, $69: 365-386$.

DANELL, R. (2000), Stratification among journals in management research: A bibliometric study of interaction between European and American journals, Scientometrics, 49 : 23-38.

GlänZEL, W., SChlEMmer, B., ThiJst, B. (2003), Better late than never? On the chance to become highly cited only beyond the standard bibliometric time horizon, Scientometrics, $58: 571-586$.

GläNZEL, W., SChUBERT, A., BrAun, T. (2002), A relational charting approach to the world of basic research in twelve science fields at the end of the second millennium, Scientometrics, $55: 335-348$.

JOKIĆ, M (2001), Časopis Tekstil u pedesetogodišnjem razdoblju od 1952. do 2000. godine - neki od bibliometrijskih pokazatelja, Tekstil, $50: 614-622$.

JOKIĆ, M. (2003), Evaluation of Croatian journals covered by the ISI databases (Institute for Scientific Information), Periodicum Biologorum, 105 : 95-98.

JOKIĆ, M.(1998), The significance of Acta Adriatica in scientific communication in the field of marine biology, fisheries and oceanography, Acta Adriatica, 39 : 81-90.

JoKić, M., ANDreis, M., KLAIĆ, B. (2002), Pedeset godina Kemije u industriji - bibliometrijski i scientometrijski prikaz, Kemija $u$ Industriji, 51 : 116-122.

Jokić, M., Sirotić, G. (2002), The communicability of the journal Acta Botanica croatica over the 1991-2000 period, Acta Botanica Croatica, $61: 221-230$.

KLAIĆ, B., ANDREIS, M., Impact factors of scientific categories - A critical analysis of SCI (1975-2002), manuscript to be submitted.

KoZlOWSKI, J., RADOSEVIC, S., IRCHA, D. (1999), History matters: The inherited disciplinary structure of the post communist science in countries of Central and Eastern Europe and its restructuring, Scientometrics, $45: 137-166$

LANGE, L. L. (2002), The impact factor as a phantom, Is there a self-fulfilling prophecy effect of impact? Journal of Documentation, $58: 175-184$

LEYDESDORFF, L., ZHOU, P. (2005), Are the contributions of China and Korea upsetting the world system of science? Scientometrics, 63: 617-630.

MARIČIĆ, S., SOROKIN, B., PAPEŠ, Z. (2000), Croatian journals at the end of the 20 century - A bibliometric evaluation, Društvena Istraživanja, $9: 1-17$.

Must, U. (2006), "New" countries in Europe - Research, development and innovation strategies vs bibliometric data, Scientometrics, $66: 241-248$.

NiSONGER, T. E. (1999), JASIS and library and information science journal rankings: A review and analysis of the last half-century, Journal of the American Society for Information Science, 50 : 1004-1019.

Ren, S., Rousseau, R. (2002), International visibility of Chinese scientific journals. Scientometrics, $53: 389-405$.

SAnZ, E., ARAgON, I., MENDEZ, A. (1995), The function of national journals in disseminating applied science. Journal of Information Science, $21: 319-323$.

SCHuBERT, A. (2002), The web of scientometrics. A statistical overview of the first 50 volumes of the journal, Scientometrics, $53: 3-20$. 
M. ANDREIS \& M. JOKIĆ: An impact of Croatian journals measured by citation analysis

Sombatsompop, N., Ratchatahirun, P., Surathanasakul, V., Premkamolnetr, N., Markpin, T. (2002), A citation report for Thai academic journals published during 1996-2000, Scientometrics, $55: 445-462$.

SOROKIN, B. (1990), Život (domaćih) časopisa u Hrvatskoj, Scientia Yugoslavica, 16 : 163-178.

Turban, E., ZHOU, D., MA, J. (2004), A group decision support approach to evaluating journals, Information \& Management, $42: 31-44$.

Ugolini, D., CAsilli, C. (2003), The visibility of Italian journals, Scientometrics, 56 : 345-355.

VAN LeEuwen, T. N., MoED, H. F. (2005), Characteristics of journal impact factors: The effects of uncitedness and citation distribution on the understanding of journal impact factors. Scientometrics, 63 : 357-371.

ZitT, M., Ramanana-Rahary, S., Bassecoulard, E. (2003), Correcting glasses help fair comparisons in international science landscape: Country indicators as a function of ISI database delineation. Scientometrics, $56: 259-282$. 